\title{
EFFECTS OF RESISTANCE TRAINING ON MUSCLE PERFORMANCE AND FUNCTIONAL CAPACITY IN BREAST CANCER SURVIVORS
}

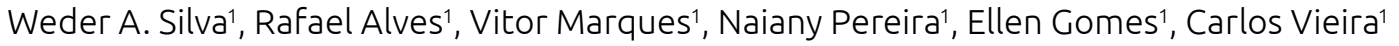

'Universidade Federal de Goiás - Goiânia (GO), Brazil.

Purpose: The aim of this study was to analyze the effects of different resistance training (RT) volumes on muscle strength and functional capacity in breast cancer survivors (BCS) undergoing hormone therapy (tamoxifen ${ }^{\circledR}$ oranastrozol). Methods: A total of 13 untrained women breast cancer survivors were randomized to one of the following TR groups: high resistance training volume (HRTV: 6 women; $57.0 \pm 8.3$ years old; height: $1.59 \pm 0.0 \mathrm{~m}$; body mass $63.7 \pm 7.61 \mathrm{~kg}$ ) or low resistance training volume (LRTV: 7 women; $55.1 \pm 7.4$ years old; height: $1.58 \pm 0.0 \mathrm{~m}$; body mass: $59.8 \pm 15.1 \mathrm{~kg}$ ). Participants performed a full-body RT routine (e.g. leg press, bench press, lat pulldown, stiff, and sit-up) once a week for 8 weeks. All exercise sets were performed until momentary muscle failure within a range of predefined repetitions in both groups HRTV (12-15 repetitions) and LRTV (6-8 repetitions), in a total of three sets for each exercise. Upper and lower limb strength was evaluated by the 10-repetition maximum test using the bench press and leg press, respectively. Functional capacity was assessed by the Timed Up and GO test. Results: Both groups showed changes in performance variables (bench press $-\mathrm{F}=36.35, \mathrm{p}<0.001$ and leg press $-\mathrm{F}=87.42, \mathrm{p}<0.001)$, and in functional capacity $(\mathrm{F}=13.84, \mathrm{p}=0.003)$, regardless of protocol used (HRTV and LRTV), with no differences between groups. Conclusion: Both protocols used in the present study (HRTV and LRTV) when prescribed in a supervised manner and in minimum weekly doses can contribute to the increase in strength of upper and lower limbs in a similar way and, consequently, can provide improvements in the functional capacity of BCS under hormone therapy treatment.

Keywords: Exercise; Hormone Therapy; Resistance Training. 